\title{
Editorial
}

\section{Antennas and RF Front Ends for Cognitive Radio}

\author{
Mohammed Al-Husseini, ${ }^{1}$ Ali El-Hajj, ${ }^{2}$ Mario Bkassiny, ${ }^{3}$ \\ Said El-Khamy, ${ }^{4}$ and Amor Nafkha ${ }^{5}$ \\ ${ }^{1}$ Beirut Research and Innovation Center, Lebanese Center for Studies and Research, Beirut 2030 8303, Lebanon \\ ${ }^{2}$ Department of Electrical and Computer Engineering, American University of Beirut, P.O. Box 11-0236, Beirut 1107 2020, Lebanon \\ ${ }^{3}$ Department of Electrical and Computer Engineering, State University of New York (SUNY Oswego), Shineman 426, Oswego, \\ NY 13126, USA \\ ${ }^{4}$ Department of Electrical Engineering, Faculty of Engineering, Alexandria University, 22 Army Street, Alexandria 21526, Egypt \\ ${ }^{5}$ Signal, Communication è Embedded Electronics Group, Supélec, 35576 Cesson-Sévigné, France \\ Correspondence should be addressed to Mohammed Al-Husseini; husseini@ieee.org
}

Received 5 May 2014; Accepted 5 May 2014; Published 4 June 2014

Copyright (C) 2014 Mohammed Al-Husseini et al. This is an open access article distributed under the Creative Commons Attribution License, which permits unrestricted use, distribution, and reproduction in any medium, provided the original work is properly cited.

The current radio spectrum regulations assign specific bands to particular services and grant licensed bands access to only licensed users. This results in the inefficient use of the frequency spectrum, a problem for which cognitive radio (CR) [1] is thought to provide a suitable solution. CR implements dynamic spectrum allocation policies by allowing unlicensed or secondary users (SUs) to access spectrum bands licensed to primary users (PUs) while avoiding interference with them. This could happen, for example, when an SU's device searches for an unoccupied band, called a white space, and uses it for its transmission. As a result of this mode of operation, significant challenges have to be dealt with when designing the RF components of a CR transceiver, such as the antennas, the filters, the mixers and oscillators, the power amplifiers (PAs), and the analog-to-digital and digital-toanalog converters (ADCs/DACs). This comes in addition to the necessary algorithms for sensing the surrounding environment and adapting to particular situations through a cognitive engine [2].

In CR, ultrawideband (UWB) antennas are usually required for channel sensing and frequency reconfigurable antennas for communicating. The two antennas can be designed in a single- or dual-port configuration. In the dualport case, one port has UWB frequency response and is used for channel sensing, and the second port, which is frequency reconfigurable/tunable, is used for communicating. In the more challenging single-port design, the same port can have
UWB response for sensing and can be reconfigured for tunable narrowband operation when required to communicate over a white space. In a distinct spectrum sharing approach, the SU device sends its transmission as UWB pulses with nulls in the bands used by PUs. In this case, the antenna should have a UWB response but with the ability to selectively induce frequency notches in the bands used by PUs. The design of UWB antennas normally requires the proper selection of the patch shape, with preferred round shapes and edges, the use of partial or slotted ground planes, the good matching between the feed and the patch, or the employment of fractals. For frequency band notching, the use of split-ring resonators (SRRs) and complementary split-ring resonators (CSRRs) is famous [3]. Frequency reconfiguration in antennas is usually attained by the use of RF and optical switches or varactors that change the paths or electrical lengths seen by the current on the radiator surface [4].

In addition to the CR antenna design challenges, the constraints on the RF front-end design pertain to the ADC/DAC sampling rate, especially when working with wideband signals, to the sensitivity of the circuitry, since the CR system has to deal with very high to very low PU signal strength, to the linearity and bandwidth of amplifiers (LNAs), and to the frequency agility of the mixer and filters [5]. Designs that guarantee the good performance of the RF components in both wideband and narrowband operation modes are required for $C R$. 
Some of the well-known spectrum sensing algorithms are energy detection and cyclostationarity-based detection. However, blind techniques, which detect the presence of a signal without any knowledge of signal or noise parameters, are more robust [6]. The spectrum sensing algorithms should be devised to overcome main sensing challenges such as RF impairments.

We hope that readers of this special issue will find up-todate information, comprehensive reviews, and robust designs for antennas and RF blocks for use in CR transceivers. We also hope that they gain a good insight into the most practical channel estimation, spectrum sensing, and decision making algorithms for cognitive radio.

Mohammed Al-Husseini
Ali El-Hajj
Mario Bkassiny
Said El-Khamy
Amor Nafkha

\section{References}

[1] J. Mitola III and G. Q. Maguire Jr., "Cognitive radio: making software radios more personal," IEEE Personal Communications, vol. 6, no. 4, pp. 13-18, 1999.

[2] S. Haykin, "Cognitive radio: brain-empowered wireless communications," IEEE Journal on Selected Areas in Communications, vol. 23, no. 2, pp. 201-220, 2005.

[3] M. Al-Husseini, K. Y. Kabalan, A. El-Hajj, and C. G. Christodoulou, "Cognitive radio: UWB integration and related antenna design," in New Trends in Technologies: Control, Management, Computational Intelligence and Network Systems, M. Joo Er, Ed., chapter 20, pp. 395-412, InTech, Rijeka, Croatia, 2010.

[4] M. Al-Husseini, K. Y. Kabalan, A. El-Hajj, and C. G. Christodoulou, "Reconfigurable microstrip antennas for cognitive radio," in Advancement in Microstrip Antennas with Recent Applications, A. Kishk, Ed., chapter 14, pp. 337-362, InTech, Rijeka, Croatia, 2013.

[5] B. Razavi, "Cognitive radio design challenges and techniques," IEEE Journal of Solid-State Circuits, vol. 45, no. 8, pp. 1542-1553, 2010.

[6] Z. Khalaf, A. Nafkha, and J. Palicot, "Blind spectrum detector for cognitive radio using compressed sensing and symmetry property of the second order cyclic autocorrelation," in Proceedings of the 7th Internationa ICST Conference on Cognitive Radio Oriented Wireless Networks and Communications (CROWNCOM '12), pp. 291-296, Stockholm, Sweden, June 2012. 

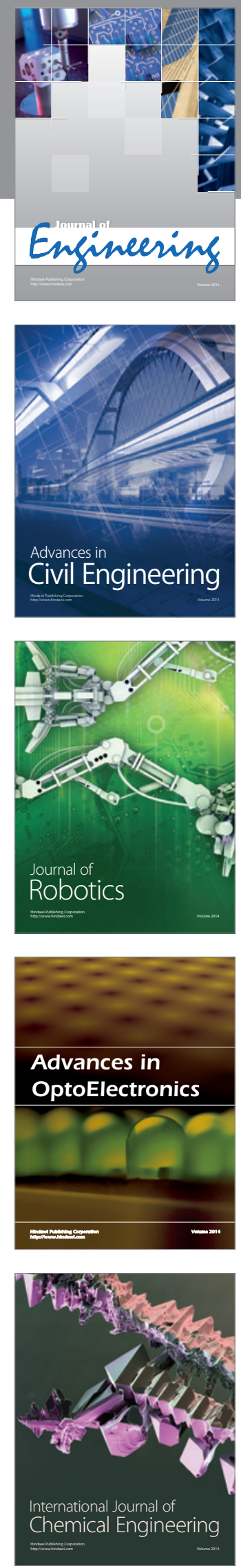

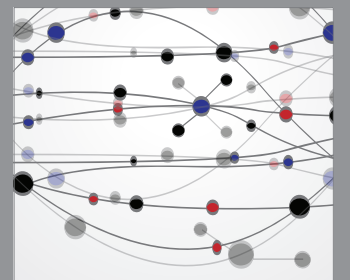

The Scientific World Journal
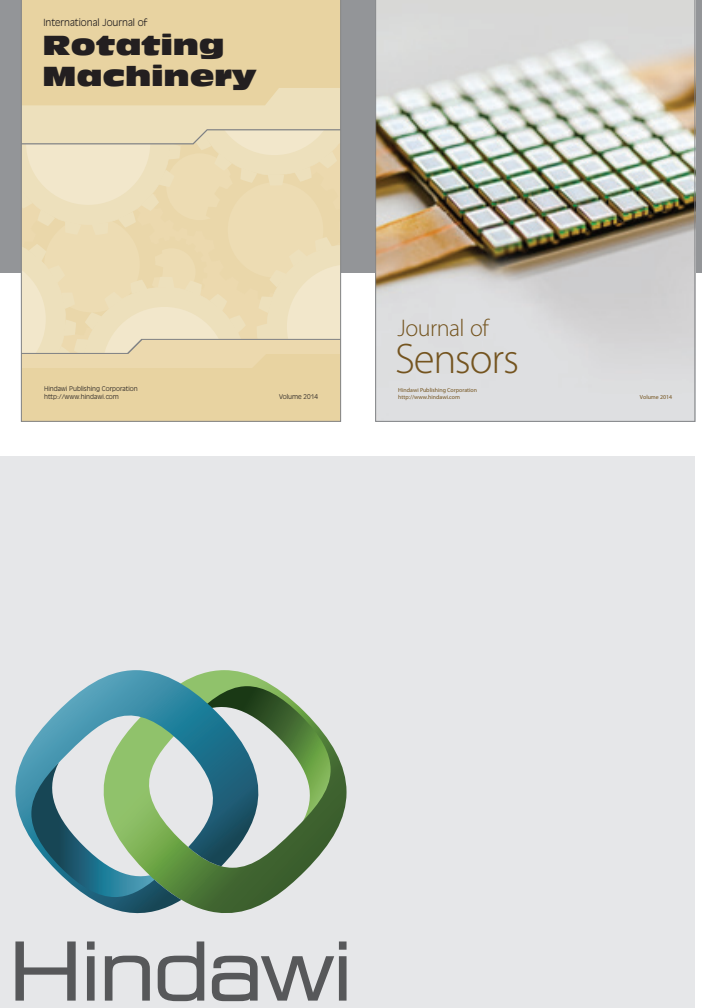

Submit your manuscripts at http://www.hindawi.com
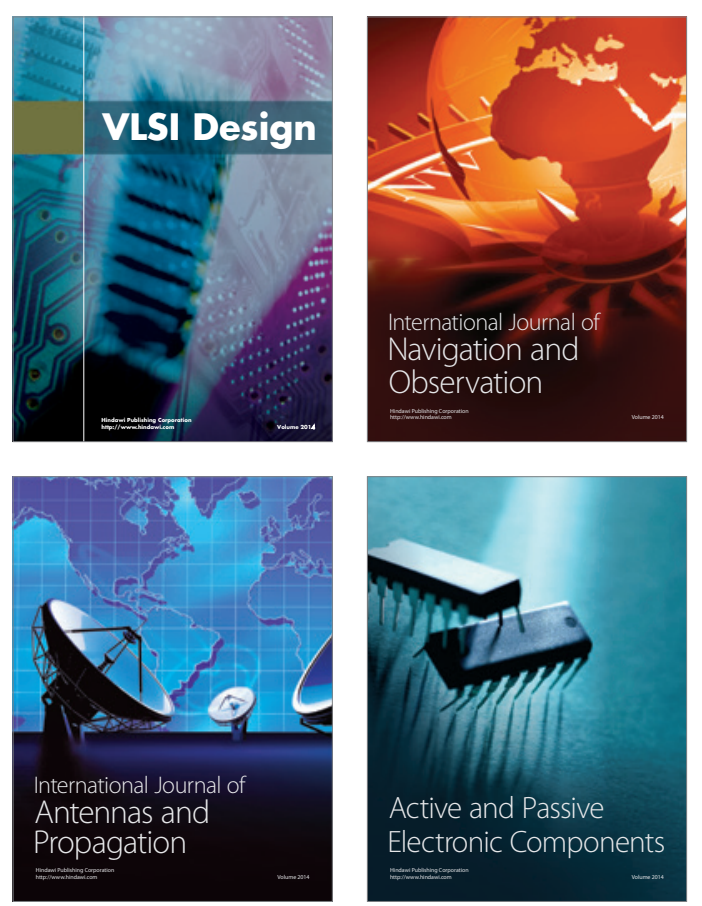
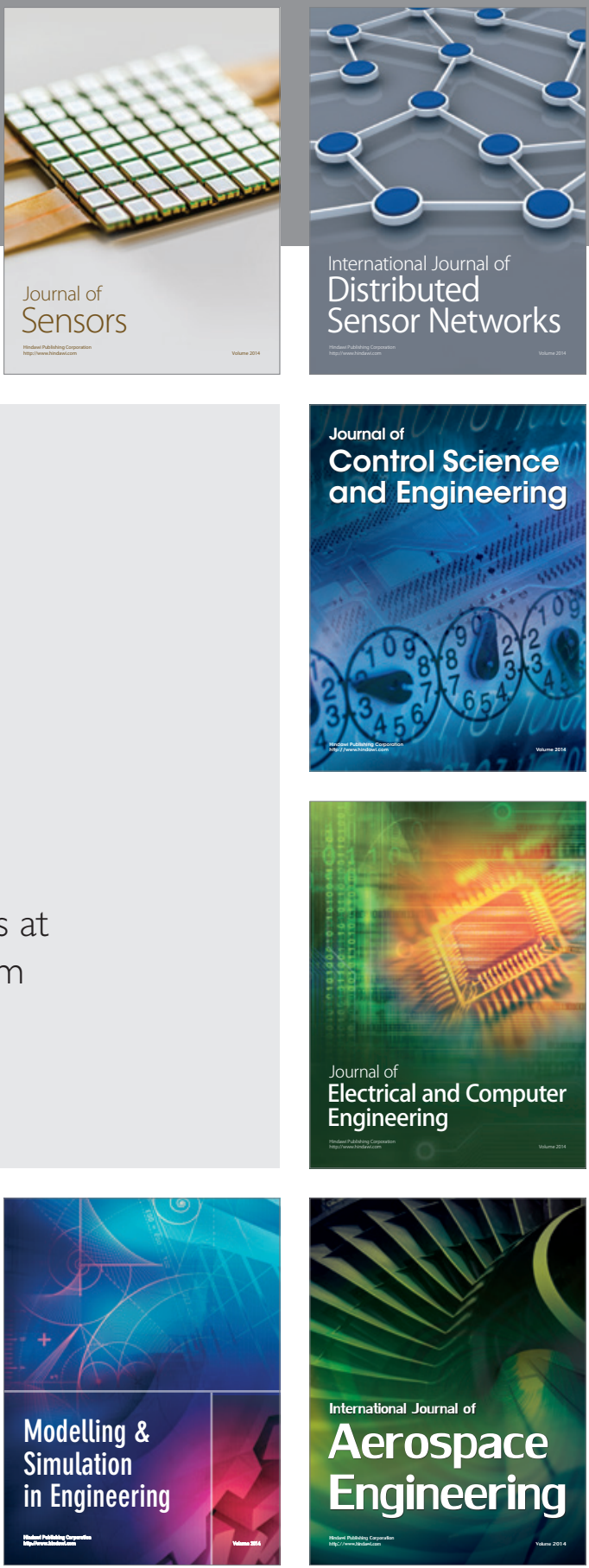

Journal of

Control Science

and Engineering
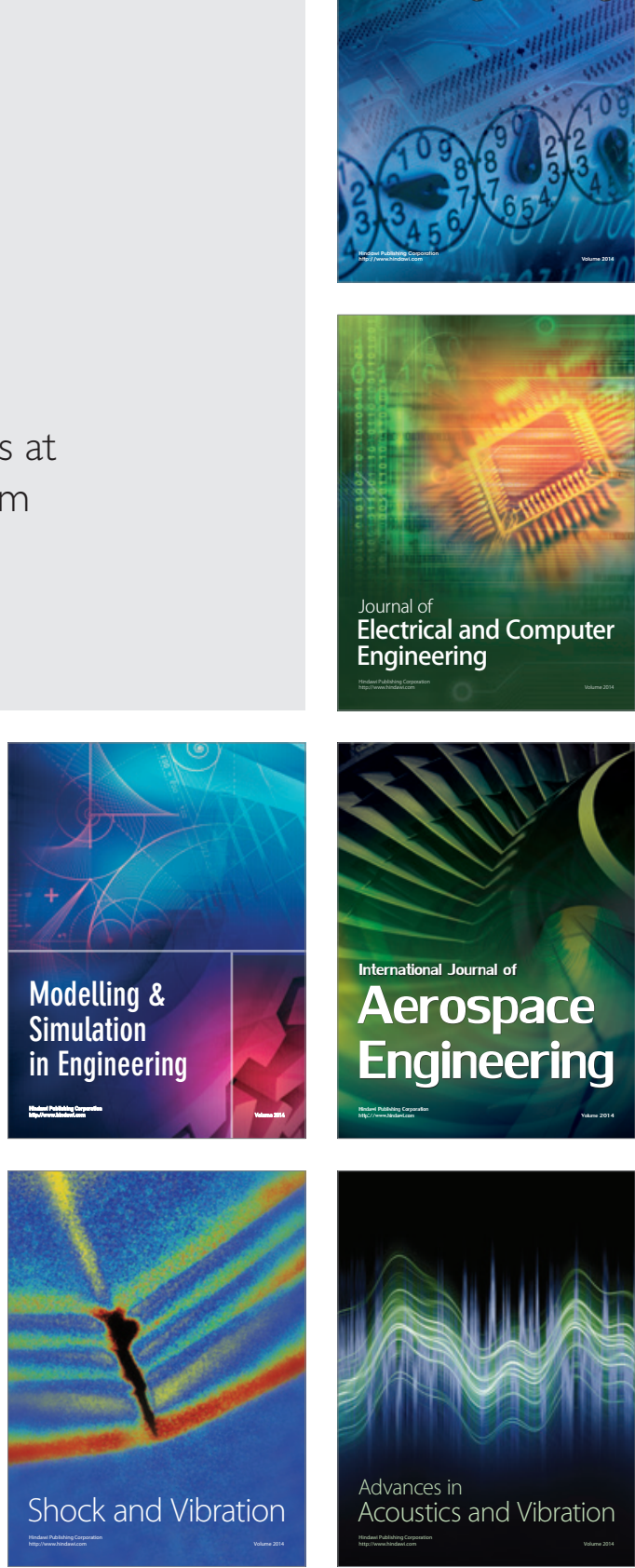